\title{
New 'stimuli-enriched' laboratory bioassay used to identify improved botanical repellent treatment, Lem-ocimum, to control the stored-grain pest Tribolium castaneum.
}

Iliyasu Mohammed Utono ${ }^{\mathrm{a}, \mathrm{b}}$, Gabriella Gibson ${ }^{\mathrm{a}}$

\author{
a. Natural Resources Institute, Chatham Maritime, University of Greenwich, ME4 4TB \\ (g.gibson@gre.ac.uk) \\ b. Institute for Agricultural Research, Ahmadu Bello University, Zaria \\ (imutono@yahoo.com)
}

Corresponding author: Dr Iliyasu M. Utono, phone +2348038381767, emailimutono@yahoo.com

\begin{abstract}
A laboratory study of Tribolium castaneum, a major pest of stored grain, was conducted to develop a more efficient and effective 'choice’ bioassay for identification of new repellent botanical treatments. Standard bioassays to test the repellency of candidate plants include pitfall traps and open arena choice tests, environments lacking in some of the most important natural stimuli that guide the movement of food-searching beetles, e.g., 1) materials they can burrow through, which stimulate 'positive thigmotaxis', 2) a range of light and dark areas, which stimulate 'negative phototaxis' and 3) three-dimensional habitats, which stimulate 'positive geotaxis.' The lack of these stimuli can lead to two common problems; 'low efficiency' (high proportion of beetles remain in the area that surrounds treatments without making a 'choice'), and 'low efficacy' (high variability in proportions found in control and
\end{abstract}


treated samples). The new 'stimuli-enriched' bioassay, which included all three of the above stimuli, was significantly more efficient (Analysis of deviance; $\chi^{2}=82.4, \mathrm{df}=3, P<0.0001$ ) and effective $\left(\chi^{2}=30.6, \mathrm{df}=3, P=0.0027\right)$ than three standard bioassays. The stimuli-enriched bioassay was used to compare the repellency of four candidate plants; Ocimum basilicum (Sweet Basil) and Cymbopogon nardus (Lemongrass) were significantly more repellent than Vernonia amygdalina or Nauclea diderrichii (Tukey Contrasts; $P<0.01$ ). A novel method of applying repellent material (a paste of repellent plant is applied between the layers of double bagged grain) was tested on the most promising repellent plants materials; a combination of C. nardus and O. basilicum ('Lem-ocimum') at $0.5 \% \mathrm{w} / \mathrm{w}$ of each was significantly more effective than $O$. basilicum on its own (Tukey Contrasts; $P<0.05$ ). These results show that the stimuli-enriched bioassay provides more consistent and accurate assessments than the standard bioassays of the repellency of candidate botanicals, and that Lem-ocimum treated double- bags are a promising new method of protecting sorghum from T. castaneum.

Keywords: Tribolium castaneum, bioassay, botanicals, repellents, geotaxis, phototaxis, thigmotaxis.

\section{Highlights}

- More efficient \& effective repellent plant bioassay was developed to protect grain.

- Tribolium beetles more responsive in the new bioassay than in the standard assays.

- Lemongrass and sweet basil were the most repellent plants in the bioassay.

- Combination of repellent plants reduced number of beetles infesting bags of grain. 


\section{Introduction}

Monitoring and control of pest insects is often based on controlling their behaviour by presenting semio-chemicals (chemical attractants or repellents) to trap, kill or repel target species. Laboratory-based bioassays have been an important tool for testing the behavioural responses of target species to candidate compounds under controlled semi-natural environmental conditions prior to field testing under natural conditions (Robertson et al., 2007); large numbers of insects can be tested against a wide range of chemicals and doses relatively quickly. In particular, bioassays have played a key role in determining the efficacy of repellent plant materials and in identifying their active ingredients against storage crop insect pests (Morgan et al., 1998; Lale \& Yusuf, 2001; Stefanazzi et al., 2011). However, the strength of response, which determines the efficacy of a bioassay, depends on the quality of the environmental stimuli present in the bioassay. Many standard bioassays test whether a material is an attractant or repellent by measuring the insect's response (positive or negative 'chemotaxis') to volatile chemicals emanating from the material and carried by a moving air current (Campbell, 2012). The movement of insects in their natural environments, however, is also controlled by responses to a wide range of stimuli; e.g., 'phototaxis' causes movement toward or away from light (Reza \& Parween, 2006), 'geotaxis’ causes movement up or down in response to gravity (Cox \& Collins, 2002; Jiang et al., 2006) and ‘thigmotaxis’ causes movement along pathways that maximise the area of their bodies in contact with surfaces (Kennedy, 1986). All of these stimuli-driven responses help beetles locate a food source and avoid contact with toxic chemicals, while keeping them in a protected environment. Hence, bioassays should incorporate the main stimuli insects respond to in specific environments, so that the outcome of the bioassay reflects what is likely to occur in the field. Otherwise, a stimulus-poor bioassay can interfere with the natural searching behaviour of beetles, and, therefore, reduce the reliability of the bioassay outcome. 
It has been observed that a major problem with Tribolium castaneum (Herbst) bioassays is that significant proportions of the beetles spend most of their time walking around the edges of the arena where they can be in contact with the floor and a wall, infrequently moving across the open area of the arena (Surtees, 1963; Yinon \& Shulov, 1969; Campbell \& Hasgtrum, 2002; Olsson et al., 2006; Duehl et al., 2011; Campbell, 2012). A study by Campbell \& Hagstrum (2002) of the behaviour of T. castaneum in a bioassay arena found that they moved across a bioassay arena more frequently if a network of walls was present throughout the arena. We tested the hypothesis that this is due, at least in part, to the strength of their response to thigmotactic cues; i.e that beetles prefer to maintain contact with substrates over as much of their bodies as possible, presumably to protect themselves from desiccation and from detection by predators (Romero et al., 2010).

In the study presented here, a new bioassay was designed to take into account phototaxis, geotaxis and a rarely considered response, thigmotaxis, with the aim of identifying promising repellents with greater efficiency and efficacy than three standard bioassays (long-drop pitfall, open arena and open arena with shallow pits). The new, stimulienriched bioassay was tested against these three standard bioassays to compare the strength of response of $T$. castaneum to sorghum grain treated with a known repellent, methyl salicylate.

The bioassay was also used to compare the response of $T$. castaneum to four commonly used repellent plants; Sweet basil (Ocimum basilicum (L.)), Lemongrass (Cymbopogon nardus (L.)), Bitter leaf (Vernonia amygdalina) (Delile), Yellow tree (Nauclea diderrichii) (De Wild \& T. Durand) Merrill and a combination of the two most repellent plants; Lemongrass (C. nardus) and Sweet basil (O. basilicum), hereafter referred to as 'Lemocimum', plants that are grown in the area of the field experiments in a laboratory in Kebbi, Nigeria. These species were chosen because there is evidence in the literature (Asawalam et 
al., 2006; Musa et al., 2009; Mishra et al., 2012) that they are repellent to a range of stored crop pests. However, information on the efficacy of these plant materials on $T$. castaneum infesting sorghum is limited, and no published information was found on the efficacy of dried powder of these plants on T. castaneum.

Finally, the bioassay was used to assess the efficacy of a new method for protecting grain from T. castaneum infestations developed by Utono (2013) in response to evidence that the conventional method of mixing repellent botanicals with grain storing in bags does not give optimum protection, as practiced by farmers in study area of Utono (2013) and the present study area (Kebbi state, Nigeria) and described by Koona et al. (2007) for use against cowpea beetles. Mixing repellent plant materials with stored grain does not reduce the ability of insects to penetrate into the bags, the repellent plant material is dispersed at a relatively low density throughout the grain, and it is time-consuming to remove the repellent plant material before preparing the grain for food or for selling (Utono, 2013). Therefore, Utono (2012) and Utono et al. (2013) developed and field-tested a 'repellent-treated double-bag' method for protecting sorghum grain based on the results of stimuli-enriched bioassays presented here. A paste of dried Lem-ocimum is applied between the layers of a double bag, and just pure grain is placed within the inner bag, with the aim of concentrating the repellent plant material in a layer surrounding the grain, and increasing the physical impedance of beetles from moving through two layers of bag to reach the grain.

The main aims of this study were to design a more efficient and effective bioassay to identify from a range of plant materials, the plants and doses with the most promising repellent effects to protect stored grain from $T$. castaneum, and to evaluate the effectiveness of a new method for applying repellent plant material to stored grain; double bags treated with a low dose of a repellent plant material to protect grain within the inner bag grain from beetle infestations. 


\section{Materials and methods}

\subsection{Experiment 1: Development of a new 'stimuli-enriched' bioassay}

A new bioassay apparatus was designed with the aim of increasing the efficiency and efficacy with which the assay measures the response of $T$. castaneum to test stimuli, as compared to standard bioassays, by incorporating a greater number of environmental stimuli that if encountered could mediate the behaviour of beetles when they search for food. In this case, an 'efficient' bioassay is defined as resulting in a high proportion of test beetles caught in either treated or untreated grain, with few wandering around in the rest of the assay arena, and an 'effective' bioassay is defined as producing the clearest difference in response to the control and treatment, i.e. the least variable results and the greatest difference in the proportions of beetles caught in the untreated and treated grain for a given dose. To assess the relative improvement of the new bioassay, the response of beetles to a standard dose of a known repellent, methyl-salicylate, was compared using three standard bioassays (pitfall trap and two versions of an open arena choice test) and the new bioassay.

\subsubsection{Standard bioassays}

Pitfall traps. 'Pitfall' type choice traps rely on the movement of beetles throughout the trap arena to bring them within detectable range of the test material, whereupon they fall into the trap if the test material is a suitable attractant or avoid the trap if it is a repellent, The pitfall trap used for this study consists of a petri dish $(9 \mathrm{~cm}$ in diameter, Alpha Laboratories UK) with two holes, placed equidistant to the sides of the dish and each other, and each hole is fitted with an eppendorf tube (1.5 ml, Alpha Laboratories UK) with the bottom cut off, such that the tops of the eppendorf tubes are level with the floor of the petri dish. Each of the eppendorf tubes is inserted into one of two centrifuge tubes (15 ml, Alpha Laboratories UK) 
underneath the petri dish (Fig. 1A). One centrifuge tube contains the treated test grain sample and the other contains the control (untreated) test sample. This apparatus allows odour from the tubes to emanate upwards into the petri dish, and beetles released in the centre of the petri dish 'choose' between the two odours.

Open arena choice test. This bioassay apparatus consists of a large open tray arena (58 cm long x $39 \mathrm{~cm}$ wide x 8.5cm deep) in which beetles can move around freely and make a choice between the control and treated grain samples, which are contained in netting bags $($ mesh size $=1.5 \times 1.5 \mathrm{~mm}$, bag $=8 \times 8 \mathrm{~cm})$ placed on the floor at either end of the rectangular tray (Fig. 1B). These bags were used because the mesh size was large enough to allow beetles to move in and out of the mounds of grain, so that the response to test materials could be evaluated independently of the response to the barrier presented by standard grain storage bags. Beetles were confined in the tray by a ring of Fluon around the rim of the tray.

\subsubsection{Modified open tray bioassays}

Open arena choice test with 'pits'. The first modification to the open arena apparatus was designed to enable natural negative phototactic and geotactic behaviour; the treated and control samples of grain are each placed in a shallow pit $(1 \mathrm{~cm}$ deep $)$ in the floor at either end of the arena (Fig. 1B), whereby $50 \%$ of the test grain samples are below floor level.

Open arena with stones and pits ('stimuli-enriched' arena). The second modification of the open arena apparatus was designed to provide an environment rich in thigmotactic (i.e. a complex of 'edges') and additional phototactic cues; the floor of the open tray is covered in a double layer of small stones (10-20 mm diameter, Garden Centre pebbles); as the beetles move through the pebbles, they maintain contact with surfaces over a greater area of their bodies and stay out of direct light for a greater proportion of the time 
than they would in an open arena (Fig. 1C). Test grain samples were placed in the pits as for the previous bioassay. This assay provided the beetles with more physical cues they use under natural conditions to search for food without ever having to go out into the open, and provided for thigmotactic, negative phototactic and geotactic behaviour. The presence of the stones may have also modified the sensory environment by altering the diffusion pattern of volatiles emanating from the test materials. The construction of the bioassay apparatus and the respective tests for efficiency and efficacy of each bioassay type were conducted in the controlled environment facilities at NRI.

\subsubsection{Positive control repellent}

To compare the efficiency and efficacy of the four types of bioassay, the grain was treated with a chemical known to be repellent to T. castaneum (methyl salicylate) as a 'positive' control, so that it was certain that the all the bioassays should demonstrate repellency (Jayasekara et al., 2005). Grain in small bags was treated with a dose of methyl salicylate that was found to be effective in deterring $T$. castaneum in our preliminary studies.

\subsubsection{Experimental protocol}

Tribolium castaneum beetles were presented with a choice of $10 \mathrm{~g}$ of whole wheat grain (Triticum aestivum L.) treated with $100 \mu \mathrm{l}$ of the repellent methyl salicylate $(10 \mathrm{mg} / \mathrm{ml})$ or $10 \mathrm{~g}$ of grain treated with $100 \mu \mathrm{l}$ of acetone as the control. Whole wheat grain was used for this experiment which was conducted at NRI because it is the grain used to maintain the $T$. castaneum colony. A relatively high dose of methyl salicylate was used compared to the effective dose found by Jayasekara et al. (2005) to be sure of a strong repellent effect when comparing different types of bioassay. The treated grain was allowed to dry for 2-3 min, $10 \mathrm{~g}$ of grain per sample was placed in netting bags and then used for the bioassay to test the 
efficacy of the treatments against $T$. castaneum. Forty, 4 days starved un-sexed, 7-10-day old beetles were introduced at the centre of the dish. Experiments were run for $4 \mathrm{~h}$ and repeated for a total of seven replicates for each bioassay type. The positions of the treatment and control grain were randomised for each replicate. At the end of each experiment the number of beetles in the treated grain, the untreated grain and remaining in the rest of the assay arena were counted and recorded, and the data were subjected to statistical analysis. The same methods of preparation of repellent materials, beetles, sample size, treatment, number of replicates, and methods of counting and recording of beetles were used as mentioned above in all the assays, unless stated otherwise.

\subsection{Experiment 2: A stimuli-enriched bioassay to assess repellency of four candidate plant} materials

Having established that the new bioassay was the most efficient and effective bioassay of the four tested in Experiment 1, we used it to assess the relative repellency of four plants that are locally available in the region of the field study and are known to be repellent to T. castaneum; Sweet basil (O. basilicum), Bitter leaf (V. amygdalina), Yellow tree ( $N$. diderrichii) and Lemongrass (C. nardus). These experiments were conducted in the laboratories of the College of Agriculture, Zuru, Nigeria. All plant materials were harvested fresh in three villages (Tondi, Maga and Wasagu) in the southern area of Kebbi state, Nigeria, shade-dried for 3-4 days, packed in polypropylene bags and stored in a relatively cool, dark place for up to 7 days prior to the start of the experiments. On the day of an experimental run, whole dry plant material was ground to a powder manually with a laboratory pestle and mortar, and weighed to the required amount based on a relatively low dose $1.0 \% \mathrm{w} / \mathrm{w}$ established to be effective in our preliminary studies. Whole sorghum grain (Sorghum bicolor (L.) Moench) was chosen as the test grain for this experiment, a) to validate the NRI results 
with wheat in the field in Kebbi State, Nigeria, and b) because sorghum is known to be a major host of T. castaneum in Nigeria (Lale \& Yusuf, 2000; Turaki et al., 2007) and in a survey of small-scale farmers in Kebbi State, sorghum was reported to be the grain that suffered the greatest proportionate loss to insect pests during storage, mostly due to $T$. castaneum (Utono, 2013). Since O. basilicum was reported to be the most commonly used repellent plant by the farmers in Kebbi State to protect their stored grain (Utono, 2013), we also tested combinations of this plant with the other main candidate repellent, C. nardus, which was shown to be repellent to T. castaneum in our preliminary experiments to test for potential synergistic effects that would increase the efficacy of plant materials as repellents.

The relative degree of repellency of each plant or combination of plants was assessed by placing one treated grain sample (20 g whole sorghum grain mixed with $1.0 \% \mathrm{w} / \mathrm{w}$ of test plant in a netting bag) and one untreated control sample (20 g whole sorghum grain in a netting bag) at opposite ends of the stimuli-enriched arena and releasing 40 beetles/replicate (as for 2.1.4 Experimental protocol) in the centre of the arena, allowing them unrestricted access to both the treated and control samples for $8 \mathrm{~h}$ per replicate, after which the number of beetles in the treated and in the un-treated bags were counted and recorded. The experiment was repeated seven times for each of the five treatments (four types of plant @ 1\% w/w and one combination of O. basilicum and C.nardus @ 0.5\% w/w of each).

\subsection{Experiment 3: The stimuli-enriched bioassay to test the efficacy of a novel method of} protecting grain with repellent plant materials

The bioassay was used to test a new approach to using plant materials to protect grain from beetle infestation without contaminating the grain with the plant material. A waterbased paste of dried, powdered repellent plant material is painted onto the outside the bags of grain. Once the paste has dried, the bag is placed inside a second bag, thereby producing a 
concentrated source of repellent odours around the grain bag and a double layer of bag around the grain to physically restrict the movement of beetle into the grain bag. This approach was expected to be an improvement over the old method used by farmers and described by Koona et al. (2007) to protect grain from legume beetles by placing the grain in a single-layer jute bag impregnated with aqueous extracts of the repellent botanical, Lantana camara L.

The experimental bags were made of material similar to that commonly used for grain storage in the field study area, Kebbi (woven polypropylene bags). For the purposes of the bioassay experiments, small bags were made from this material by sewing together $10 \mathrm{x} 10$ cm squares cut from larger polypropylene bags. To test the efficacy of repellents independent of the physical barrier, a preliminary test was undertaken to determine how porous the bags needed to be to ensure beetles could enter the bags reasonably unhindered. It was found that removal of $\sim 25 \%$ of the woven threads enabled $\sim 40 \%$ of the beetles to enter the control bags within the standard $8 \mathrm{~h}$ duration of the bioassay. It was decided that this was sufficient to enable a reasonable comparison of the additional repellent effect of adding candidate plant materials to of these loosely-woven double bags.

The repellent materials tested included the two species of plant materials grown locally in Kebbi that were found to be most repellent in stimuli-enriched bioassays; $O$. basilicum and C. nardus. A relatively low dose $(0.5 \% \mathrm{w} / \mathrm{w})$ of a water-based paste of each plant type was made from their dried and powdered leaves, and a third paste was made by mixing the powder of both plants together ( $0.5 \%$ w/w of each), to produce 'Lem-ocimum' paste.

The small experimental grain storage bags were filled with $200 \mathrm{~g}$ of whole wheat grain, tied up with string and painted with one of the three treatment pastes; O. basilicum, $C$. 
nardus or Lem-ocimum. These treated bags were allowed to dry for $10 \mathrm{~h}$ before inserting them into a second bag.

The degree of protection these treatments provided against beetle damage was assessed by placing one treated bag and one control bag (double-bag of grain with no repellent plant material added) at opposite ends of the stimuli-enriched arena and releasing 40 beetles/replicate (as for 2.1.4 Experimental protocol) in the centre of the arena, allowing them unrestricted access to both the treated and control bags for $8 \mathrm{~h}$, after which the number of beetles in the treated and in the un-treated bags were counted and recorded. The experiment was repeated seven times for each of the three treatments. An additional 'control' treatment consisted of placing a bag of untreated grain at each end of the bioassay arena, and comparing the proportion of beetles in the two untreated bags.

\subsection{Insect culture}

A culture of $T$. castaneum maintained at NRI was used for the bioassay at NRI and a culture maintained at Ahmadu Bello University, Zaria insectary was used for the bioassays in Nigeria. All the cultures were reared on a mixture of wheat flour and yeast in the ratio 50:5 by weight. This mixture was placed in a 2.5 l glass jar along with 100 unsexed adults of $T$. castaneum. The upper neck inside the surface of the jar was coated with Fluon and the jar was sealed with filter paper gummed with paraffin wax to prevent the beetles from escaping. After 25 days, the newly emerged adult beetles and the parent stock that were still alive were removed and disposed of. Five days later, newly emerged adults were collected every three days thereafter for use in the experiments. The newly emerged adults were collected using forceps and transferred to a new jar with no food and starved for four days before they were used in a bioassay test. The cultures were maintained and the bioassays were conducted in a controlled environment room at $26 \pm 3^{\circ} \mathrm{C}, 67 \pm 5 \% \mathrm{RH}$, with a light regime of $16: 8$ light: dark. 


\section{Statistical analysis}

To test the effect of treatments in the bioassay tests, the results were subjected to an Analysis of Deviance using a Generalised Linear Model (GLM), with binomial errors and a logit link function. Multiple comparisons between treatments were conducted from the GLM model using the Tukey contrasts protocol (Hothorn, et al., 2008). An ANCOVA model of GLM, with binomial errors, was used to test the effect of dose on the repellency of plant treatments. The statistical tests were run using the ' $R$ ' statistic software package ( $R$ Development Core Team, 2012).

\section{Results}

4.1 Experiment 1: Comparison of the response of beetles to standard bioassays and the new stimuli-enriched bioassay

The results in Fig. 2 compare the 'efficiency' of the four bioassay methods tested; i.e., the proportion of the total number of beetles released in each replicate of the assay that were collected in the arena (hence, made no choice) instead of in either the treatment or control bags. The results show that generally the proportion of beetles making 'no choice' decreased as the bioassay method increased in complexity of the environment, from the standard bioassays (Pitfall and Open arena) to the new stimuli-enriched bioassay (Open arena with pits and stones). The results of the GLM analysis of deviance indicate a significant effect of type of bioassay on the proportion of beetles that made no choice $\left(\chi^{2}=82.4, \mathrm{df}=3\right.$, $P<0.001)$. A significantly greater proportion of beetles made no choice in the open arena $(0.45 \pm 0.029$; mean \pm standard error $)$ and pitfall trap $(0.37 \pm 0.033)$ than in the open arena with pits (0.26 \pm 0.027$)$ or the stimuli-enriched bioassays $(0.12 \pm 0.0197$, Tukey contrasts; $P<0.001$ in all cases). This suggests that most modifications to the standard bioassays improved the 
efficiency of the bioassays; $~ 88 \%$ of test beetles made a choice in the new bioassay, against only 55- $63 \%$ in the two most standard bioassays.

Figure 3 shows the comparison in 'efficacy' of the four bioassays; i.e., the proportion of beetles caught in treated grain of those beetles that made a choice (i.e. were caught in either treated or untreated grain). The proportion below 50\% caught in treated grain can be taken as a measure of the repellency of the treated grain. These results show that there was a gradual increase in the proportion of beetles repelled by the treated grain with increasing complexity of the bioassay environment, from the pitfall bioassay (on the left of plot) to the stimuli-enriched bioassay (on the right of plot). The analysis of deviance indicates a significant effect of 'type of bioassay' on the proportion of beetles repelled by treated grain $\left(\chi^{2}=30.62, \mathrm{df}=3, P=0.0027\right)$ and the Multiple comparisons tests show a significantly lower proportion of beetles were found in treated grain in the stimuli-enriched bioassay $(0.19 \pm 0.024)$ than in the open arena bioassay $(0.27 \pm 0.356)$ or in the pitfall trap bioassay (0.34 \pm 0.041 , Tukey contrasts; $(P<0.001$ in all cases). There was no significant difference $(P=0.485)$ in the mean proportion of beetles found in treated grain between pitfall and open arena or open arena with pits. The addition of pits and stones increased the difference in proportion of beetles in treated grain by $12-16 \%$, thereby increasing the accuracy of the assay.

4.2 Experiment 2: The new stimuli-enriched bioassay to assess repellency of four candidate plant materials

The results in Fig. 4 show that dose (0, 0.25, 0.5 to $1 \%$ w/w) had a highly significant effect on the response of beetles to five treatment plant materials; $V$. amygdalina, $N$. diderrichii, O. basilicum, C. nardus and a combination of O. basilicum and C. nardus (Lemocimum). The results of the GLM ANCOVA indicate that overall the proportion of beetles 
found in treated grain samples decreased significantly with dose $\left(\chi^{2}=245, \mathrm{df}=1, P<0.0001\right)$. Plant type, however, had a significant effect $\left(\chi^{2}=304, \mathrm{df}=5, P<0.0001\right)$, which is reflected by the differences in slope between plant types. This interaction between dose and type of plant materials was also significant $\left(\chi^{2}=39, \mathrm{df}=4, P<0.0001\right)$, as can be seen by the greater effect of dose for Lem-ocimum than for the other plants.

The results in Fig.5 show that at the highest dose tested $(1 \% \mathrm{w} / \mathrm{w})$ for all the plant materials tested singly, $C$. nardus was the most repellent, with only a small proportion of beetles $(0.06 \pm 0.0074)$ found in the treated grain, followed by O. basilicum with $0.15 \pm 0.022$. However, the combination of plants in Lem-ocimum produced the greatest repellent effects on T. castaneum (0.02 \pm 0.0074$)$. Nauclea diderrichii and $V$. amygdalina were least repellent, with $0.27 \pm 0.027$ and $0.28 \pm 0.028$ proportion of beetles in the treated grain, respectively. The GLM analysis of deviance shows that 'type of plant' had a significant effect on the proportion of beetles caught in the treated grain $\left(\chi^{2}=45.2, \mathrm{df}=4, P<0.0001\right)$ and the Multiple comparisons tests show that the difference between the means of each treatment were significant for all the treatments ( $P<0.001$, Tukey contrasts) except between $V$. amygdalina and $N$. diderrichii $(P=0.998)$ and $C$. nardus and Lem-ocimum $(P=0.0661)$. These results suggest that $O$. basilicum, $C$. nardus and more importantly a combination of both (Lemocimum) have promising potential for improving small-scale farmers’ methods of grain protection.

\subsection{3 Experiment 3: Efficacy of treated double-bags to protect grain from insects}

The results of an experiment to determine if adding a low dose of plant materials in between layers of a double bag containing grain could reduce the chances of beetles penetrating into the bag are presented in Fig. 6. This approach involves two independent 
factors affecting access to the grain; the repellency of the plants and the physical barrier of the double-bag.

The results of the GLM analysis of deviance indicate a significant effect of treatment type on the proportion of beetles that penetrated into the treated bags $\left(\chi^{2}=19.6, \mathrm{df}=3\right.$, $P<0.0001)$. A significantly lower proportion of beetles penetrated into bags treated with Lemocimum $(0.07 \pm 0.021)$ compared to O. basilicum $(0.27 \pm 0.025)$ or the control bag $(0.49 \pm 0.038)$ (Tukey Contrasts; $P<0.05)$. Similarly, there were significant differences $(P<0.01)$ between the mean proportion of beetles found in bags treated with $C$. nardus and the control bag, but not between bags treated with $C$. nardus and Lem-ocimum $(P=0.151)$. These results suggest that the movement of beetles into treated bags was affected more by $C$. nardus than the $O$. basilicum, with a tendency for combining both (Lem-ocimum) to be the most effective.

\section{Discussion}

\subsection{The new 'stimuli-enriched bioassay' bioassay}

The ability of a particular bioassay to measure efficiently and effectively the response of insects to a test stimulus depend on the kind of orientation cues available and their ability to influence the test insects, thereby facilitating a positive response. Therefore, the purpose of this study was to determine if modifications to standard bioassays could lead to an improved bioassay that is more efficient and effective in identifying repellents.

This was confirmed by the results of Experiment 1 conducted with T. castaneum exposed to four types of bioassay arenas which differed in their environmental structures to determine the effect of enriched sensory stimuli on the response of beetles to the test stimuli. In their study of the response of $T$. castaneum to an arena with a patchy environment, consisting of a smooth floor arena with patches of grain, Campbell \& Hagstrum (2002) observed that a greater number of $T$. castaneum were found inactive in corners and edges of 
the arena than in the patches of grain, a behaviour which Kennedy (1986) termed thigmotaxis. If there are no objects in the arena, insects sometimes stop moving around, or become limited to following the edges of the arena without ever moving across open spaces toward the target treatments. This behaviour can obscure the response of insects to the test material and consequently limit the efficiency of a bioassay. It might be as a result of this limitation that even in the presence of an airflow containing attractive odours Olsson et al. (2006), Duehl et al. (2011), and Campbell (2012) observed a low response rate of $T$. castaneum to pheromone or food attractants in their bioassays.

In the present study four types of bioassays were tested that differed in their stimuli and physical features, which presented different orientation cues and resulted in different responses in the beetles; 1) the pitfall assay relied on beetle movement in an open arena to encounter the test grain, 2) the open arena assay also relied on beetle movement in an open arena to bring beetles nearer the source, and in both cases, the main areas of the arenas were flat, so many beetles stayed near the edges of the arenas and did not search the whole area, 3) the open arena assay with pits relied on similar behaviour, but the presence of positive geotaxis and negative phototaxis responses appear to have stimulated beetles to move down into the untreated grain samples once they had found them, thereby staying in the grain samples longer and 4) the open arena with pits and stones (stimuli-enriched assay) included features that stimulated positive geotaxis, negative phototaxis and positive thigmotaxis responses.

The bioassay results indicate that only about $55-63 \%$ of the 40 beetles released in standard bioassays contributed to the assessment of candidate repellents, the rest never made a choice between treatments, whereas $88 \%$ of released beetles in the stimuli-enriched bioassay made a choice between the control and treatment grain sample. The addition of each component provided more physical cues, thereby increasing the efficiency of the assay. 
The reduction in the proportion of beetles making no choice and the increase in the proportion caught in grain samples (treated and untreated) in the new bioassay could be due to a number of factors. The addition of stones all over the floor of the arena was thought to give the beetles a more continuous touch stimulus, which enhanced the amount of time they moved around the whole area of the arena, thereby reducing the chances of the beetles to gather around the edges of the tray and increased the chances of detecting odour cues from the control and treated grain; a trend observed also by Campbell \& Hagstrum, (2002). In the study presented here, in the open arena and pitfall arena a greater proportion of beetles was observed to walk continuously around the edge of the arena trays or the pitfall petri dishes, to hide behind the netting bags used to contain the grain samples or gather in the corners of the tray in the open arena assays. In the open arena assay the beetles were observed to hide under the netting bags, consistent with the observation of Romero et al. (2010) that beetles have a preference for remaining sheltered, hence putting the bottom half of the netting bags of grain into the sunken pits allowed the beetles to shelter and remain in the pits even if the beetles had not been attracted or repelled by odours emitted from the bag of grain.

Although we have alluded to a few reasons why the sensory-enriched environment may have led to the increased efficiency and efficacy of the sensory-enriched bioassay, further studies are required to establish the precise mechanisms involved. It is possible that multiple sensory cues interact to enhance the response to volatile cues, or simply enhance the likelihood that beetles come within range of detection of treated samples, or spend more time within the vicinity of treated samples.

It is also important to bear in mind that bioassays should be appropriate for the natural context they are intended to imitate. In the case of the present study, the aim was to determine which type of repellent might be most effective at reducing beetle infestation. To investigate the efficacy of attractants for surveillance traps that are placed in the open, for example, on 
the floor of a store-room, bioassays would benefit from a different range of features. When bioassays lack the relevant environmental conditions that are required for a particular context, it is likely a high proportion of insects will not fully respond in the bioassay, and hence, extra replicates and experimental materials are required to obtain a significant result. A bioassay that is efficient and effective gives better outcomes by presenting beetles with the appropriate cues during the bioassay. This point has been demonstrated by the results of the study presented here.

The laboratory experiment conducted with four types of plant (O. basilicum, $N$. diderrichii, V. amygdalina and C. nardus) proved that the method practiced by farmers to protect their stored sorghum grain with ground dry plant materials can be effective against $T$. castaneum. However, differences in efficacy experienced by the farmers could be related to the specific plant materials and methods of application used.

Nigerian specimens of $C$. nardus and $O$. basilicum were significantly more repellent than $V$. amygdalina or $N$. diderrichii. Significant repellency of V. amygdalina and $N$. diderrichii was found only at the highest dose tested $(1 \% \mathrm{w} / \mathrm{w})$ and it even appeared to be mildly attractive to the beetles at the lowest dose tested (0.25\% w/w, Fig. 4). Surprisingly, no literature was available on the repellency of these two plants for T. castaneum except that the major compound in V. amygdalina, 1.8-cineole, was reported to provide moderate repellency against T. castaneum by Obeng-Ofori \& Rechmuth (2009). However, it could be that the Nigerian grown $V$. amygdalina is low in concentration of the major compound, which may be the reason for its ineffectiveness at low doses. However, V. amygdalina was reported to be repellent to the beetle Callosobruchus maculatus F. (Musa et al., 2009) and Sitophilus zeamais Motschulsky (Asawalam \& Hassanali, 2006). More research is required to make conclusions on the repellent efficacy of these plants for T. castaneum. 
The high repellency exhibited by the dried ground leaves of $C$. nardus combined with O. basilicum is likely to be related to their respective chemical compositions. Mikhaiel (2011) and Mishra et al. (2012) reported that essential oils from both O. basilicum and C. nardus are known to possess compounds that repel T. castaneum. Even though the two plants were both repellent to $T$. castaneum individually, C. nardus was significantly more repellent than O. basilicum, which suggests that either the two plants possess different concentrations of the same compounds or different compounds that affect $T$. castaneum differently. The response exhibited by $T$. castaneum to the plant materials suggests that repellency is dose dependant and repels beetles more effectively at higher doses.

Since the two most repellent plants were found to differ in their efficacy we tested for synergism by combining them at a low dose $(0.5 \% \mathrm{w} / \mathrm{w})$. Harris (2002) reported that combinations of two or more control materials can be synergistic and improve the efficacy of the combined products. Synergistic effects are important in pest management because they entail the use of low doses, and combinations of materials can lead to products with multiple modes of action (Harris, 2002), hence, reduced chances for pest resistance to treatments developing. A combination of powdered Nicotiana tobacum L. Tagetes minuta L., Tephrosia vogelii L. and Azadirachta indica A. Juss was found to be more effective in reducing plant damage and mortality by the bean bruchid Acanthoscelides obtectus Latreille than the use of N. tobacum alone (Agona \& Muyinza, 2003).

Evidence reported here indicates that a combination of $O$. basilicum and $C$. nardus may have stronger repellent properties than the individual plants on their own. Although $C$. nardus was not significantly more repellent than Lem-ocimum, the repellency of $O$. basilicum was certainly enhanced by mixing it with $C$. nardus. This synergistic effect is important and indicates that the two plants can be used together to develop a simple and effective method to control this pest in the field requiring less plant material overall, which 
would overcome one of the factors that limits the successes of repellent plants as control materials (Isman, 2006). However, the greater challenge is to identify how farmers can best treat their grain with this combination of plant repellents to improve protection for their stored grain. The method of mixing grain with repellent plant material to be stored in polypropylene bags is practiced by small-scale farmers of Kebbi, but often with little success, and, therefore, there is high demand for improved methods of protecting grain with repellent plants.

\subsection{Effect of adding plant material between layers of polypropylene double bags on beetle} infestation

The farmers’ method of mixing grain with powdered plant material or plant extract leaves unwanted residues or increases the labour and time required for processing the grain before it can be used or sold. Some plant materials used in grain protection are reported to be toxic (Isman, 2006) and the methods used by farmers may not ensure a total removal of residues (Belmain et al., 2001). The long-term aim of the project is to develop a new technology based on using materials farmers already use to protect their grain, but with a greater efficacy. It is hoped that the new method will overcome the main limitations of the farmers' existing methods and enhance their confidence in and acceptance of repellent plant materials for pest control. The main idea is to use the standard double bagging method that provides a greater physical barrier to beetle infestations than single bags, but also to add repellent plant materials between the two bags, with the grain in the inner bag remaining uncontaminated by repellent plant materials. The results of this study have shown that $T$. castaneum is affected by the presence of Lem-ocimum in the treated bags and avoided it, hence the proportion of beetles that entered the treated bags was significantly reduced. 
This research highlights that by adding more natural features to standard bioassays our understanding of insect responses to the test stimuli is much improved, beetles are able to differentiate more clearly between treated and untreated grain and repellent-plant treated double bags appear to have the potential for protecting farmers' grain stored in bags. A recent field trial of Lem-ocimum treated double-bags (Utono et al., 2014) has validated the results reported here and shown that after 5 months in storage, the percent change in sorghum grain weight and levels of infestation by the two most prevalent pests, $T$. castaneum and Rhyzopertha dominica, inside Lem-ocimum treated double-bags were significantly reduced, even when only $4 \%$ of the grain in the store-rooms was kept in treated double-bags $(P<0.01$, $\mathrm{n}=120$ store-rooms).

Protecting grain with a double bag and a concentrated layer of repellent plant material surrounding the inner bag has never been tested before. Previous work on treating stored grain with repellents either by admixed grain with plant materials or treated bags with plant extract or oils, both of which resulted in low efficacy (Othira et al., 2009). In the double-bag method presented here, only a relatively small dose of plant material is required, and there is no need for time-consuming winnowing to remove the plant residues before using or selling the grain. However, the effect of the technology on long term storage is yet to be evaluated. Clearly, the next step would be to undertake a larger-scale field trial.

\section{Acknowledgements}

The authors thank the Kebbi State Government, Nigeria for the studentship support for this research, Stephen Young (NRI) for statistical advice and helpful comments from a referee. 


\section{References}

Agona, J.A. and Muyinza, H., 2003. Synergistic potential of different botanical combinations against bean bruchids in storage. African Crop Science Society 6, 216-219.

Asawalam, E.F. and Hassanali, A., 2006. Constituents of the essential oil of Vernonia amygdalina as maize weevil protectants. Tropical and Sub-tropical Agro-ecosystems 6, 95-102.

Belmain, S. R. and Stevenson, P., 2001. Ethnobotanicals in Ghana: reviving and modernising age-old farmers practice. Pesticide Outlook 12, 233-238.

Campbell, J.F., 2012. Attraction of working Tribolium castaneum adults to traps. Journal of Stored Products Research 51, 11-12.

Campbell, J.F. and Hagstrum, D.W., 2002. Patch exploitation by Tribolium castaneum: movement patterns, distribution and oviposition. Journal of Stored Product Research 38, 55-68.

Cox, P.D. and Collins, L.E., 2002. Factors affecting the behaviour of beetle pests in stored grain, with particular reference to the development of lures. Journal of Stored Products Research 38, 95-115.

Duehl, A.J., Arbogast, R.T. and Teal, P.E., 2011. Age and sex related responsiveness of Tribolium castaneum (Coleoptera: Tenebrionidae) in novel behaviour bioassays. Environmental Entomology 40, 82-87.

Harris, R., 2002. Synergism in the essential oil world. International Journal of Aromatherapy 12, 179-186.

Hothorn, T., Bretz, F. and Westfall, P., 2008. Simultaneous inference in General Parametric Models. Biometrical Journal 50, 346-363. 
Isman, M.B., 2006. Botanical insecticides, deterrents, and repellents in modern agriculture and an increasingly regulated world. Annual Review of Entomology 51, 45-66.

Jayasekara, T.K., Stevenson, P.C., Hall, D.R. and Belmain, S.R., 2005. Effect of volatile constituents from Securidaca longepedunculata on insect pests of stored grain. Journal of Chemical Ecology 31, 303-313.

Jiang, F., Jayas, D.S. and White, N.D.G., 2006. Vertical movement of adult rusty grain beetles, Cryptolestes ferrugineus in stored corn and wheat at uniform moisture content. Journal of Insect Science 6, 1-9.

Kennedy, J.S. 1986. Some current issues in orientation to odour sources. In: Payne, T.L., Birch, M.C. and Kennedy, C.E.J. (Eds.). Mechanisms in insect olfaction. Oxford: Clarendon Press, pp11-26.

Koona, P., Tatchago, V. and Malaa, D., 2007. Impregnated bags for safer storage of legume grains in West and Central Africa. Journal of Stored Products Research 43, 248-251.

Lale, N.E.S. and Yusuf, B.A., 2000. Potential of varietal resistance and Piper guineense seed oil to control infestation of stored millet seeds and processed products by Tribolium castaneum (Herbst). Journal of Stored Product Research 37, 63-75.

Mikhaiel, A.A., 2011. Potential of some volatile oils in protecting packages of irradiated wheat flour against Ephestia kuehniella and Tribolium castaneum. Journal of Stored Product Research 47, 357-364.

Mishra, B.B., Tripathi, S.P. and Tripathi, C.P.M., 2012. Repellent effect of leaves essential oils from Eucalyptus globulus (M) and Ocimum basilicum (L) against two stored grain insect pests of coleopterans. Nature and Science 10, 50-54.

Morgan, C., Sherington, J., Gudrups, I. and Bowden, N.S., 1998. The assessment of potential attractants to beetle pests: improvements to laboratory pitfall bioassay methods. Journal of Stored Product Research 34, 59-74. 
Musa, A.K., Oyerinde, A.A. and Owolabi, F.O., 2009. Evaluation of the efficacy of mixed leaf powders of Vernonia amygdalina L. and Ocimum gratissimum Del. against Callosobruchus maculatus (F.)(Coleoptera: Bruchidae). Academic Journal of Entomology 2, 85-87.

Obeng-Ofori, D., Reichmuth, C.H., Bekele, J. and Hassanali, A., 2009. Biological activity of 1,8 cineole, a major component of essential oil of Ocimum kenyense (Ayobangira) against stored product beetles. Journal of Applied Entomology 121, 237-243.

Olsson, P.O.C., Ryne, C. Wallen, R., Anderbrant, O. and Lofstedt, C. 2006. Male-produced sex pheromone in Tribolium confusum: behaviour and investigation of pheromone production locations. Journal of Stored Products Research 42, 173-182.

Othira, J.O, Onek, L.A., Deng, L.A. and Omolo, E.O., 2009. Insecticidal potency of Hyptis spicigera preparations against Sitophilus zeamais (L.) and Tribolium castaneum (Hebrst) on stored maize grains. African Journal of Agricultural Research 4, 187-192.

R Development Core Team, 2012. R: A language and environment for statistical computing. R Foundation for Statistical Computing, Vienna, Austria. ISBN 3-900051-07-0, URL http://www.R-project.org.

Reza, A.M.S. and Parween, P., 2006. Differential preference of colored surface in Tribolium castaneum (Herbst). Invertebrate Survival Journal 3, 84-88.

Robertson, J.L., Russell, R.M., Preisler, K.H. and Savin, N.E., 2007. Bioassays with arthropods (2 ${ }^{\text {nd }}$ Ed.), CRC Press (Taylor and Frances Group), USA, 193pp.

Romero, S.A., Campbell, J.F., Nechols, J.R. and With, K.A., 2010. Movement behaviour of red flour beetle: Response to habitat cues and patch boundaries. Environmental Entomology 39, 919-929.

Stefanazzi, N., Stadler, T. and Ferrero, A., 2011. Composition and toxic, repellent and antifeeding deterrent activity of essential oils against the stored-grain pests Tribolium castaneum and Sitophilus oryzae. Pest Management Science, 67, 639-646. 
Surtees, G., 1964. Laboratory studies on dispersion behaviour of adult beetles in grain. VI. Three-dimensional analysis of dispersion of five species in a uniform bulk. Bulletin of Entomological Research 55, 161-170.

Utono, M.I., 2013. A novel approach to control stored sorghum beetle Tribolium castaneum (Coleoptera: Tenebrionidae) in small-scale farmers' storerooms in Kebbi- Nigeria. PhD. theses submitted to University of Greenwich, United Kingdom. 298pp.

Utono, I.M., Coote, C. and Gibson, G. (2014) Field study of the repellent activity of 'Lemocimum'-treated double bags against the insect pests of stored sorghum, Tribolium castaneum and Rhyzopertha dominica, in northern Nigeria. Journal of Stored Products Research 59, 222-230.

Yinon, U. and Shulov, A., 1969. Bioassay of the response of Tribolium castaneum to repellent substance excreted by Trogoderma granarium. Entomologia Experimentalis et Applicata 12, 191-205. 


\section{A}

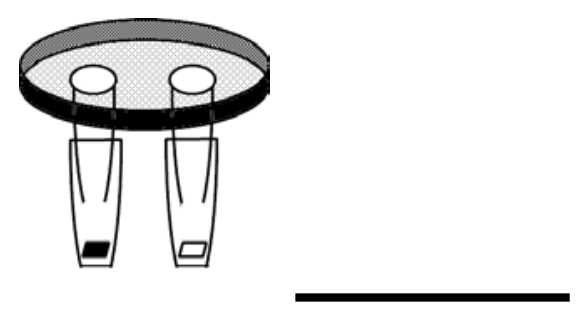

B

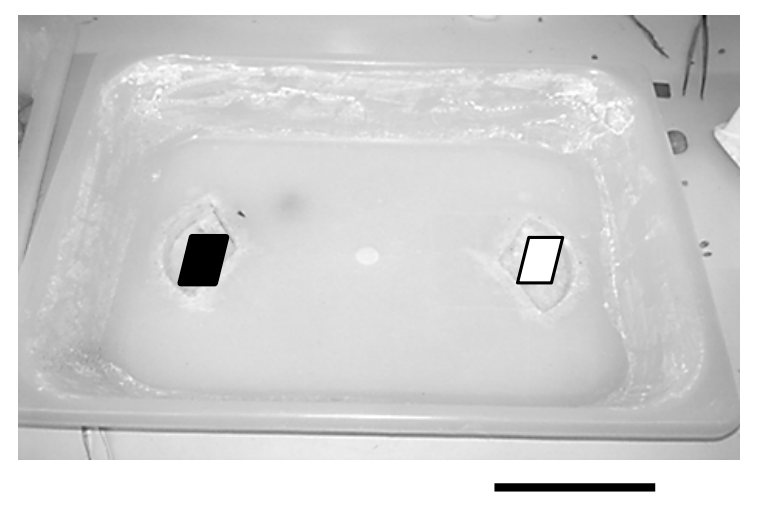

C

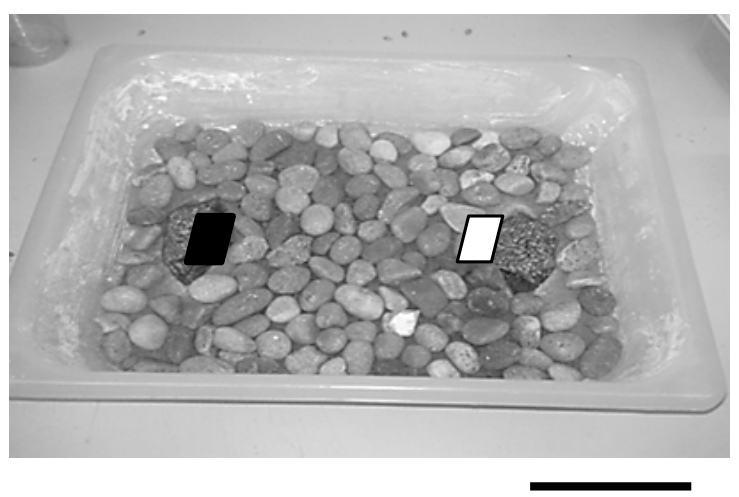

Figure 1 Bioassay types tested in Experiment 1; showing positions of treatment (black square) and control samples (white square) in each apparatus. A) Pit-fall bioassay; beetles released in centre of pertri dish at the top of the apparatus. Test grain samples placed at bottom of their respective tubes. B) Open tray arena (32 cm x $26 \mathrm{~cm}, 6 \mathrm{~cm}$ deep) showing faint outline of 'pits' into which test grain samples are placed to allow negative phototaxis and geotaxis; beetles placed in centre of tray at beginning of experiment. C) Open tray arena modified with addition of pits and stones to provide continuous 'edges' to allow thigmotaxis. Sides of tray treated with silicon paint to prevent beetles from escaping. Scale bars $10 \mathrm{~cm}$. 


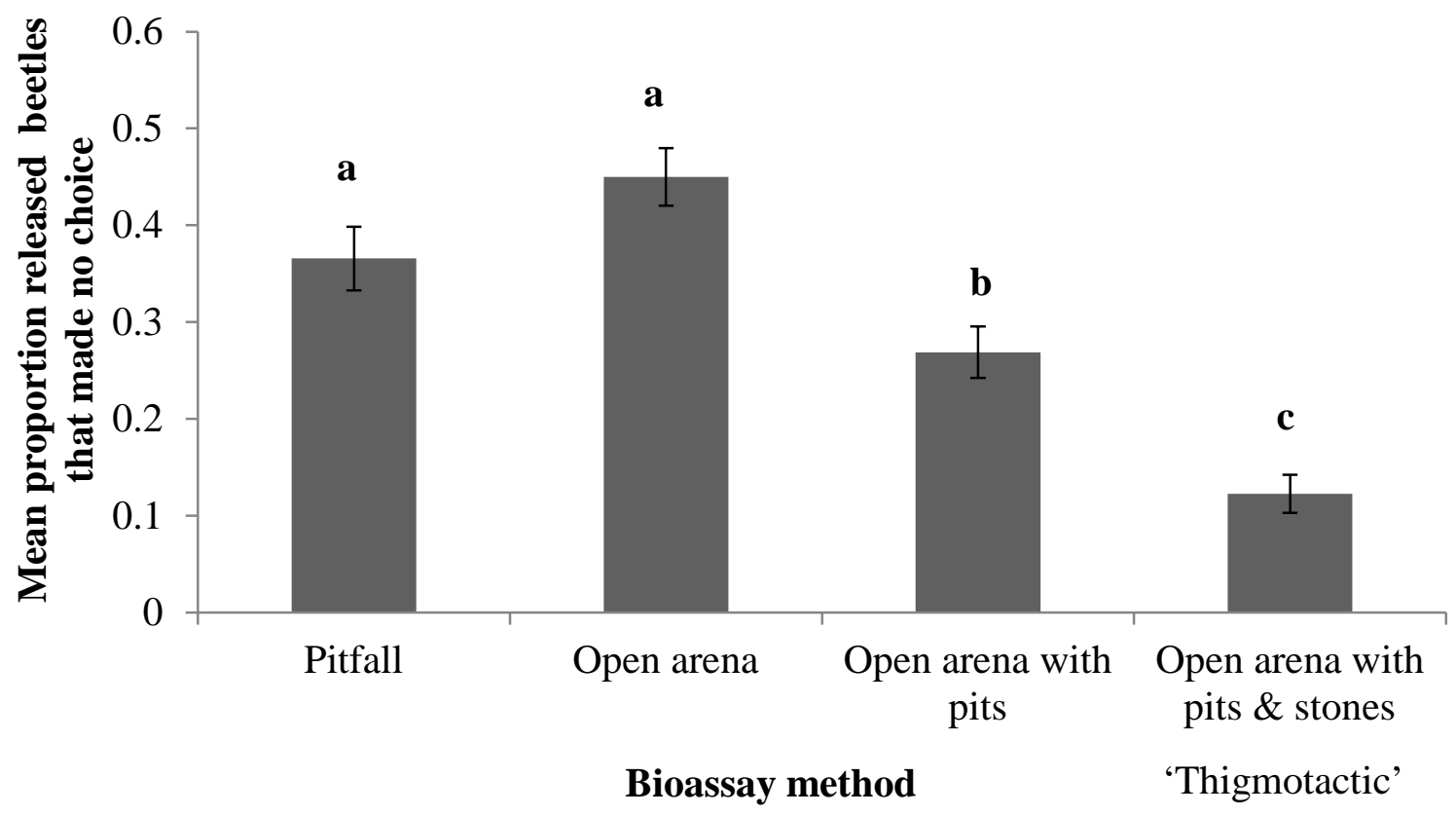

Figure 2 Comparison of the 'efficiency' of four types of repellent bioassays (Experiment 1); mean proportions $( \pm \mathrm{SE})$ beetles that were not in either the treated $(100 \mu \mathrm{lof} 10 \mathrm{mg} / \mathrm{ml}$ methyl salicylate) or untreated grain (control) samples (i.e. made no choice), out of total number beetles $(n=40)$ released in each replicate. For each bioassay type, $n=7$ replicates. Bioassay type had a significant effect on proportion of beetles that made no choice (GLM, binomial errors, Analysis of Deviance, $\left.\chi^{2}=82.4, \mathrm{df}=3, P<0.0001\right)$. Bars with different letters are significantly different $(P<0.001$, Multiple comparisons from GLM model, Tukey Contrasts). Standard errors calculated from analysis of deviance model. 


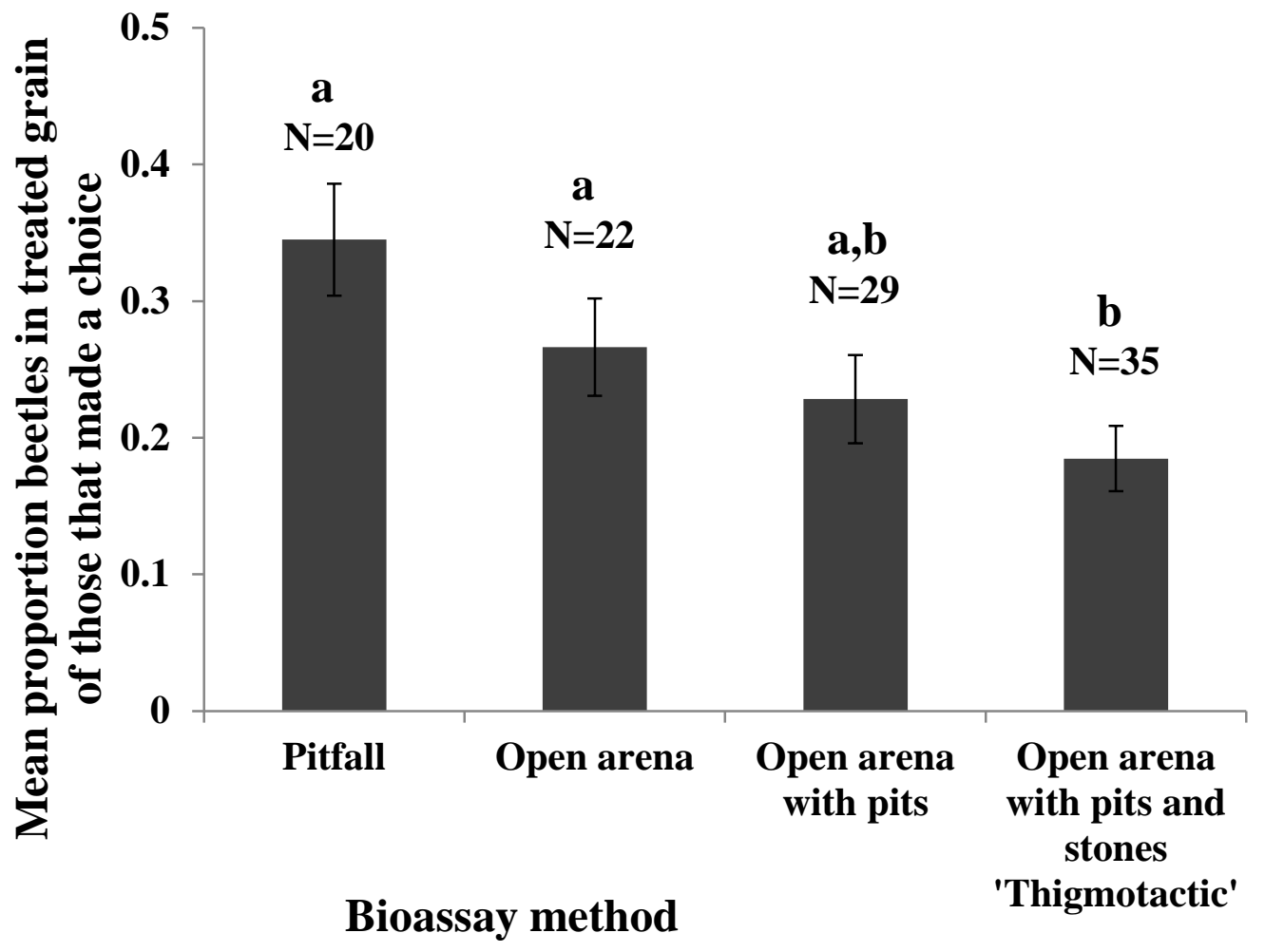

Figure 3 Comparison of the 'efficacy' of four types of repellency bioassays (Experiment 1); mean proportions $( \pm S E)$ of beetles caught in $10 \mathrm{~g}$ wheat grain samples treated with a known repellent, methyl salicylate $(100 \mu \mathrm{l}$ of $10 \mathrm{mg} / \mathrm{ml})$ of total number of beetles caught in either the treated or untreated grain (i.e. made a choice) out of 40 beetles released for each replicate. The mean number of beetles in each replicate varied (see N's above). For each 'bioassay type', $\mathrm{n}=7$ replicates. Type of bioassay had a significant effect on the proportion of beetles caught in treated grain (GLM, binomial errors, Analysis of Deviance, $\chi^{2}=30.6, \mathrm{df}=3$, $P=0.0027)$. Bars with different letters are significantly different $(P<0.001$, Multiple comparisons from GLM model, Tukey Contrasts). Standard errors calculated from analysis of deviance model. 


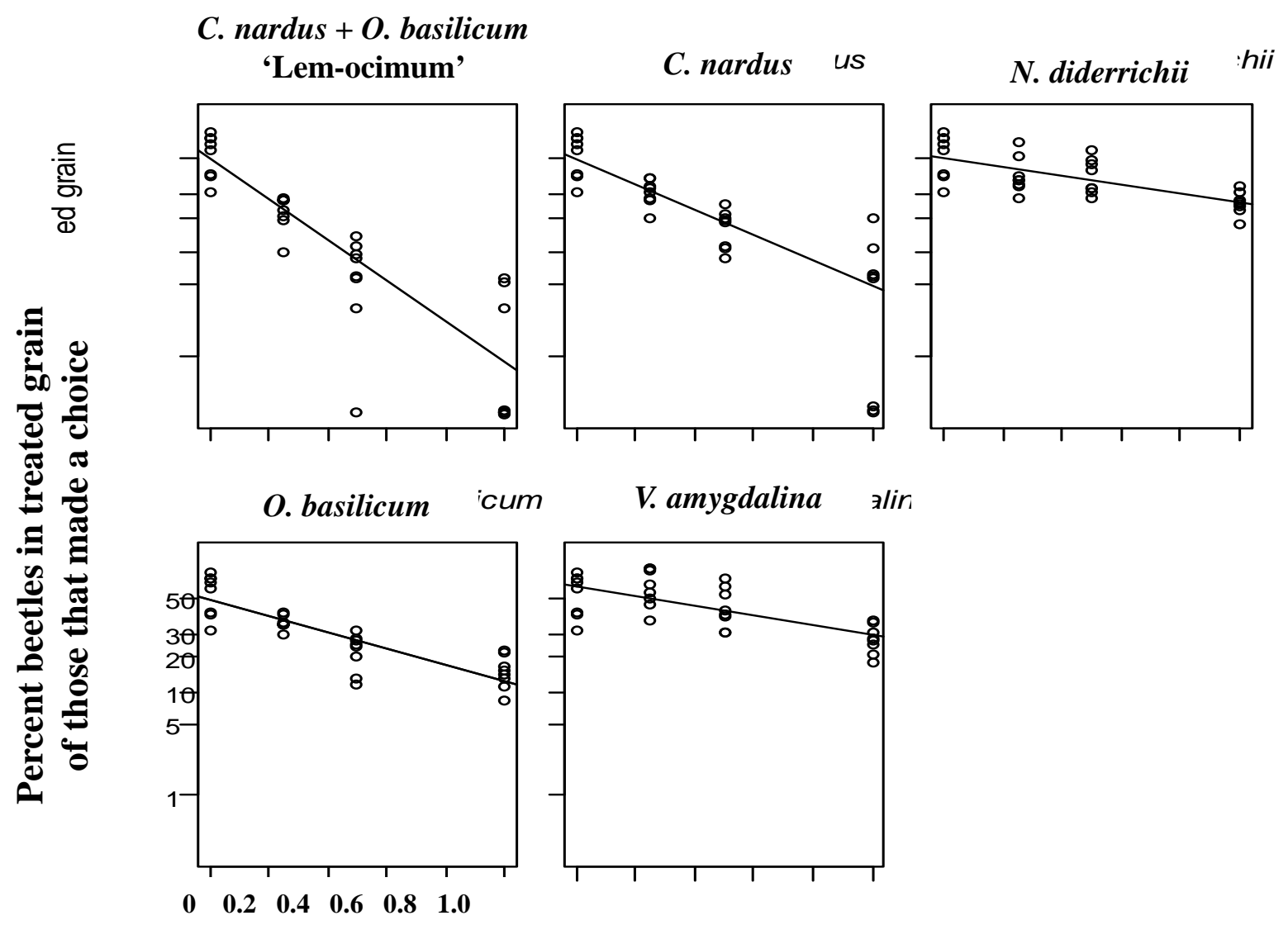

Dose (\% weight test plant material/weight grain sample)

Figure 4 Relationship between dose of test plant materials and proportion of $T$. castaneum caught in treated grain (Experiment 2); concentrations = 0, 0.25, 0.5 and 1\% w/w of 20 g samples of sorghum grain, $\mathrm{N}=40$ beetles/replicate, $\mathrm{n}=7$ replicates for each plant material and each dose. Lines are predictions from GLM ANCOVA with binomial errors. Effects of dose, plant material and their interactions are significant $\left(\chi^{2}=245, \mathrm{df}=1, \mathrm{P}<0.0001\right.$, $\chi^{2}=304, \mathrm{df}=5, \mathrm{P}<0.0001, \chi^{2}=39, \mathrm{df}=4, \mathrm{P}<0.0001$, respectively). 


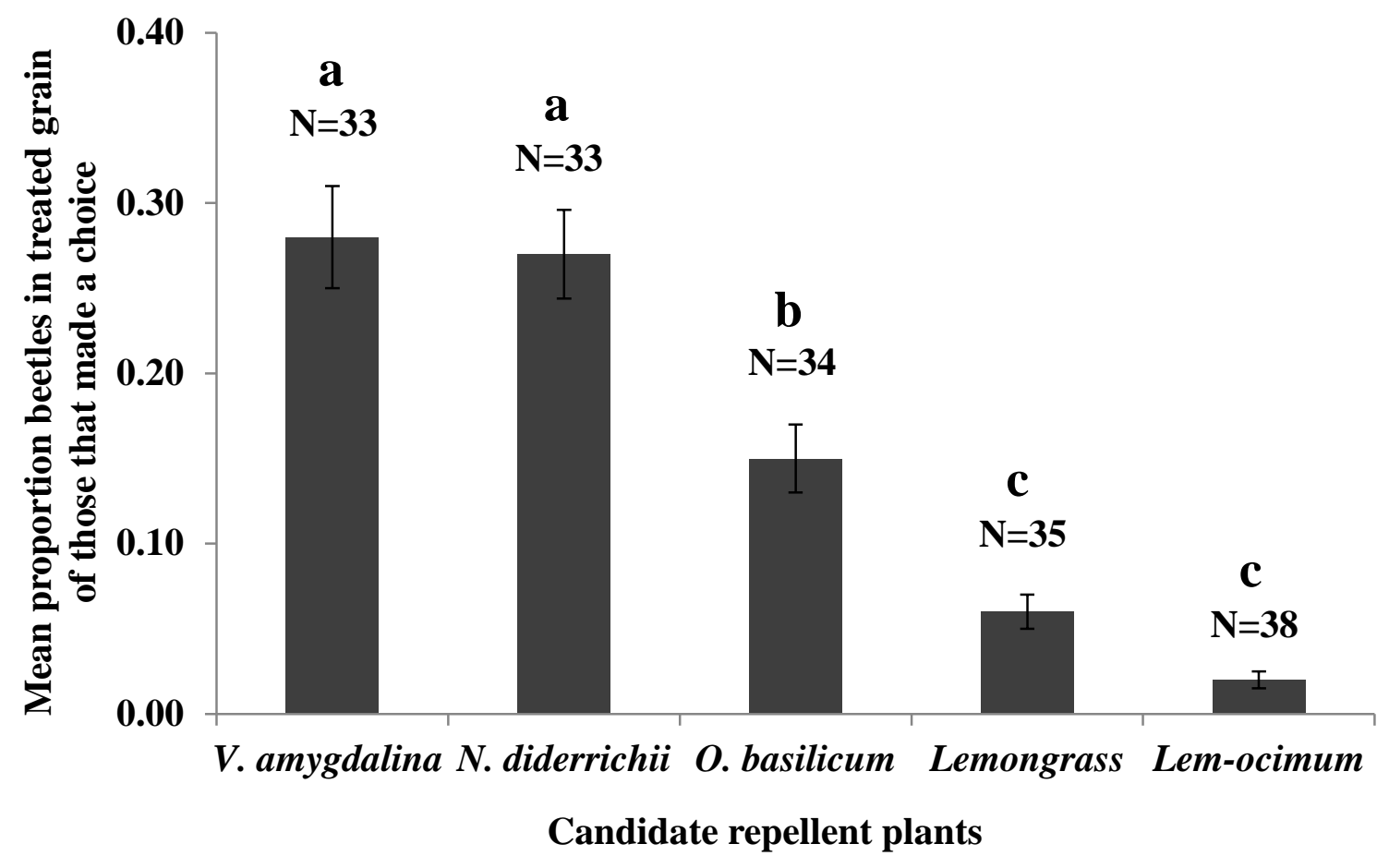

Figure 5 Thigmotatic bioassay to test relative repellency of various plant material preparations at a dose of $1 \% \mathrm{w} / \mathrm{w}$ of plant material to grain (Experiment 2); $\mathrm{N}=$ mean number beetles that made a choice between treated and untreated grain per replicate, out of 40 beetles released, $n=7$ replicates for each plant type. 'Type of plant' had a significant effect on proportion beetles caught in treated grain samples (GLM, binomial errors, Analysis of Deviance, $\chi^{2}=45.2, \mathrm{df}=4, P=0.0001$ ). Bars with different letters are significantly different $(P<0.001$, Multiple comparisons from GLM model, Tukey Contrasts). Standard errors calculated from analysis of deviance model. 


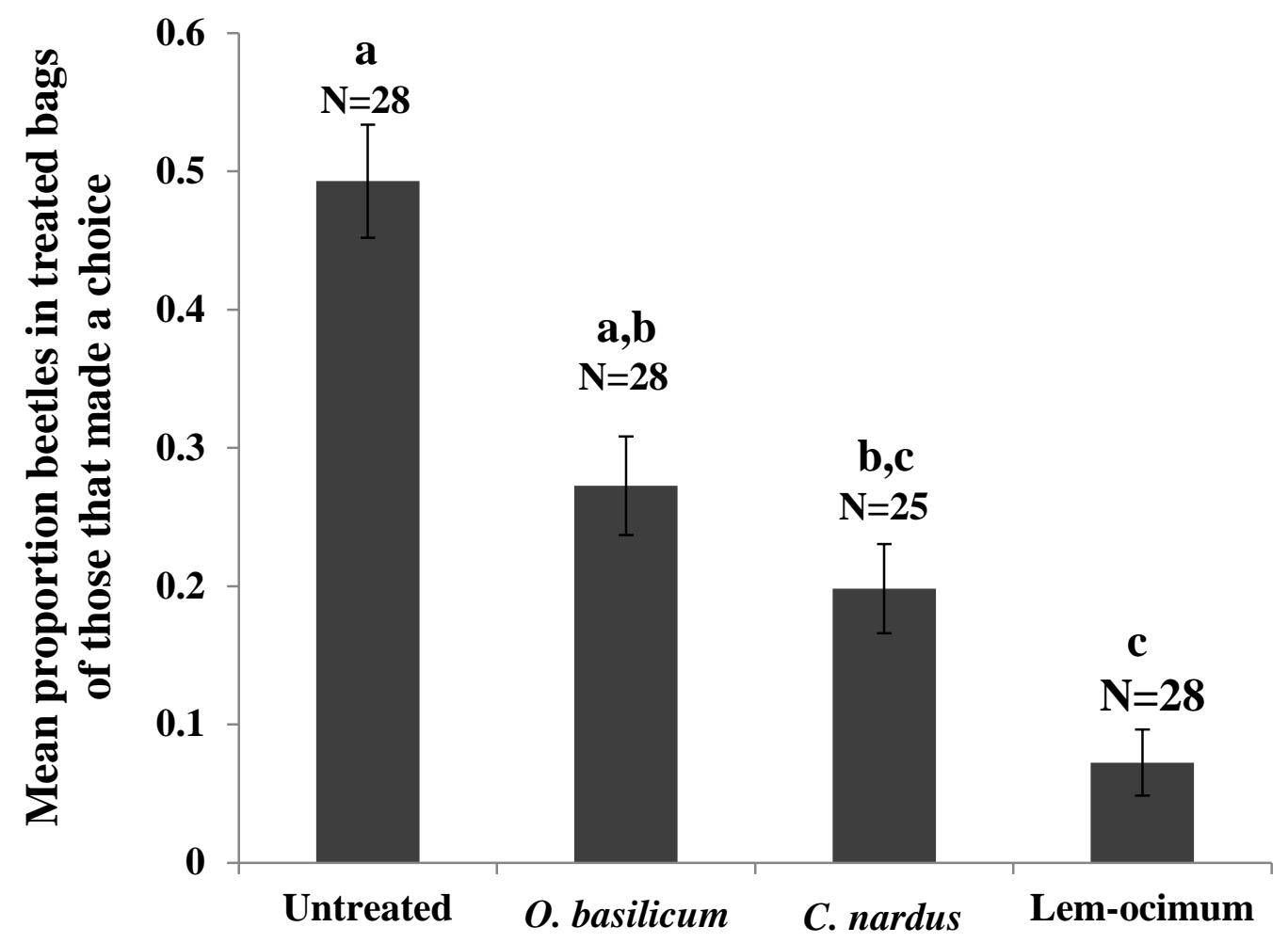

Candidate repellent plants

Figure 6 Comparison of the mean proportion ( \pm SE) beetles caught in $200 \mathrm{~g}$ samples of sorghum in double bags treated with $0.5 \% \mathrm{w} / \mathrm{w}$ of dried plant materials versus untreated control bags (Experiment 3); $\mathrm{N}=$ mean number beetles that made a choice between treated or untreated grain, out of 40 beetles released per replicate, $n=7$ replicates for each treatment. 'Type of treatment' had a significant effect on the proportion beetles caught in treated grain samples (GLM, binomial errors, Analysis of Deviance; $\chi^{2}=19.6, \mathrm{df}=3$, $P=0.0001)$. Bars with different letters are significantly different $(P<0.05$, Multiple comparisons from GLM model, Tukey Contrasts). Standard errors calculated from analysis of deviance model 